\title{
Tips and Tricks for Characterizing Shape Memory Wire Part 5: Full-Field Strain Measurement by Digital Image Correlation
}

\author{
B. Reedlunn ${ }^{1}$, S. Daly ${ }^{1}$, L. Hector, Jr. ${ }^{2}$, P. Zavattieri ${ }^{3}$, and J. Shaw ${ }^{4}$ \\ 1 Department of Mechanical Engineering, University of Michigan, Ann Arbor, MI \\ 2 Chemical Sciences and Materials Systems Laboratory, General Motors R\&D Center, Warren, MI \\ 3 School of Civil Engineering, Purdue University, West Lafayette, IN \\ 4 Department of Aerospace Engineering, The University of Michigan, Ann Arbor, MI
}

Keywords

Digital Image Correlation, Strain, Fatigue, Material Behaviors

\section{Correspondence}

B. Reedlunn,

Department of Mechanical Engineering, University of Michigan, 2350 Hayward St., Ann Arbor, MI 48109, USA

Email: breedlun@umich.edu

Received: August 25, 2010; accepted: November 29, 2010

doi:10.1111/j.1747-1567.2011.00717.x

\begin{abstract}
This is the fifth paper in a series on the experimental characterization of shape memory alloy (SMA) wires. In this installment we focus on the use of digital image correlation (DIC) to measure the strain field on the surface of the wire. After a brief overview of the principles and mathematics behind DIC, two different thermo-mechanical tension tests using DIC are presented to demonstrate the technique. The first experiment consists of Joule heating a shape memory (SM) wire to induce the shape memory effect, using 2-D DIC to measure the strain field. The second experiment measures the response of a superelastic (SE) wire to mechanical cycling at room temperature, using 3-D DIC to measure the strain field and an infrared camera to measure the temperature field. In addition to describing the experimental results, attention is paid to specimen preparation and the two experimental setups. Many of the challenges and precautions associated with using DIC are discussed, along with practical recommendations for specimen speckle patterns, digital photography, and data post processing.
\end{abstract}

\section{Introduction}

This is the fifth paper in our series, providing recommendations for the thermo-mechanical characterization of shape memory alloy (SMA) wire. Part 1 provided an introduction to the martensitic transformations that are responsible for the shape memory (SM) effect and superelasticity (SE), and demonstrated the calorimetry and thermomechanical responses of two typical NiTi SMA alloys (SM wire with austenite start temperature $A_{\mathrm{S}}>20^{\circ} \mathrm{C}$

Editor's Note: This ET feature series is intended as an introduction to this exciting area of experimental mechanics. It aims to increase awareness of active materials and to promote their consistent characterization by disseminating best practices from leading researchers in the field. Each article in the series will address the characterization of one commercially significant active material. Series editors: Nilesh D. Mankame and Paul W. Alexander. and SE wire with austenite finish temperature $\left.A_{\mathrm{f}}<20^{\circ} \mathrm{C}\right){ }^{1}$ Part 2 reviewed potential methods to obtain fundamental sets of isothermal mechanical responses. ${ }^{2}$ Part 3 highlighted stress-induced strain localization and phase-front propagation that can occur during uniaxial tension SE. ${ }^{3}$ Part 4 provided details on the thermo-mechanical coupling effects in the superelastic responses of SMA wire that cause loading-rate and ambient media sensitivities. ${ }^{4}$

One of the techniques for full-field strain measurement mentioned in Part 2 was digital image correlation (DIC). Here, we will discuss how to apply DIC measurement to the characterization of SMA wire. This is a powerful technique that gives the full-field strain distribution across a specimen, unlike conventional extensometry that gives a strain averaged over a single gage length. We will start with some background and fundamentals of DIC, describe the setup and specimen preparation, and 
then show results from two experiments using somewhat different setups, one on preconditioned SM wire and one on SE wire.

\section{Digital Image Correlation Fundamentals}

Digital image correlation (DIC) is a noncontact optical method used to measure full-field displacements on the surface of an object by tracking the nonuniform random surface patterns of a specimen. The technique is fast, robust, scalable, and provides an accurate method to determine the surface geometry, displacements, and strains of a deforming object. See Refs. 5 and 6 for further reading. It is particularly useful to quantify strain fields that are distinctly nonuniform, such as those arising from stress concentrations, and geometric or material heterogeneities/discontinuities. (Note that standard DIC algorithms are not well suited to sharp strain or displacement discontinuities, such as kinks or cracks, but this is being overcome via new techniques. ${ }^{7,8}$ ) For our case of uniaxially loaded SMA wire, large, but finite, strain gradients exist due to phase transformation.

The DIC method was first developed in the early 1980s by researchers at the University of South Carolina. ${ }^{9-11}$ Initially, DIC was created to measure in-plane deformations of flat surfaces using a single camera (two-dimensional [2-D] DIC). As the methodology developed and computing power grew over the years, the technique was improved and expanded to three-dimensional (3-D) DIC in the late 1980s and early 1990s, using two or more synchronized cameras to track the 3-D position of points on nonplanar objects (developed by McNeill, Luo, Faugeras, Chao, Sutton, Helm, and others ${ }^{12-14}$ ). Both 2-D and 3-D DIC methods calculate surface displacements and their gradients, from which surface Lagrangian strains, velocities, accelerations, and strain rates can be calculated. (Note that 3-D DIC should not be confused with volumetric DIC, where displacements and other quantities inside the material are measured, often using X-ray tomography, ${ }^{5,15}$ or confocal microscopy. ${ }^{16}$ ) The basic principles behind the DIC method have no inherent length scale or time scale. Practical limitations on the use of DIC are imposed by the accuracy of measurement techniques at smaller length scales and high speeds, but substantial progress has been made in expanding the limits of DIC as better microscopy and highspeed photography instrumentation have become available. Although 3-D DIC has obvious advantages, 2-D DIC remains a very useful and cost-effective tool to measure in-plane deformations of nominally planar samples.

The basic concept underlying DIC involves comparing two digitized images of a specimen surface, taken at different times, to obtain a quantitative, pointby-point mapping of the surface deformation. The surface deformation is obtained by optimizing a crosscorrelation function to match the local gray-scale intensity values of a reference image and a subsequent image of the deformed specimen. Commonly, a sequence of images is captured and correlated to obtain the evolution of the object's surface deformation with time. The reference image is usually taken to be the first image of the undeformed specimen to calculate the total displacement and Lagrangian strain between it and any later deformed configuration. Alternatively, to aid correlation adjacent pairs of images in the sequence can be compared, and the reference image is updated in time to give incremental displacements and strains between it and the next image.

The specimen surface, either covered by a natural or an artificially applied pattern, is digitized into a spatial distribution of pixel intensities to apply a correlation function. The images are not compared pixel by pixel, but rather by matching local distributions of pixel intensity subsets between reference and deformed images. For simplicity, the following describes how 2-D DIC is performed. Summarizing sections of Chapter 5 in Sutton et al., ${ }^{5}$ the optimal match between subsets can be achieved by minimizing a chosen correlation function, $S$, such as the simple sum of squared differences criterion,

$$
S=\sum_{i=1}^{I}\left[G_{i}-F_{i}\right]^{2},
$$

where $F_{i}=F\left(\mathbf{x}_{i}\right)$ represents the gray-scale value at pixel $i$ at position $\mathbf{x}_{i}=\left(x_{i}, y_{i}\right)$ in the reference image, and $G_{i}=G\left(\mathbf{x}_{i}^{*}\right)$ represents the gray-scale value at pixel $i$ at position $\mathbf{x}_{i}^{*}=\left(x_{i}^{*}, y_{i}^{*}\right)$ in the deformed image. The summation ranges from the first $(i=1)$ to the last pixel $(i=I)$ in the subset. Frequently this criterion is improved by adjusting for lighting variations between the reference subset and current subset. If a subset moves between areas of different lighting intensities, say from an area of high illumination to shadow, the gray-level values should scale uniformly. Thus, we can replace $G$ with $b G$ in Eq. 1 , where $b$ is a scaling parameter. The optimum value of $b$ can be found for each subset by minimizing $S$ with respect to $b$, by 
calculating

$$
\begin{aligned}
\frac{\partial S}{\partial b} & =2 \sum_{i=1}^{I}\left[b G_{i}-F_{i}\right] G_{i}=0, \\
& \Rightarrow \quad b_{\mathrm{opt}}=\frac{\sum_{i=1}^{I} F_{i} G_{i}}{\sum_{i=1}^{I} G_{i}^{2}} .
\end{aligned}
$$

Substituting $b_{\text {opt }} G_{i}$ for $G_{i}$ in Eq. 1, yields the normalized sum of squared differences,

$$
S=\sum_{i=1}^{I}\left[\frac{\sum_{j=1}^{J} F_{j} G_{j}}{\sum_{j=1}^{J} G_{j}^{2}} G_{i}-F_{i}\right]^{2}
$$

At this point, it appears that a pixel in the reference image can be matched to a location in the current image only to within a pixel; however, subpixel resolution is achieved by interpolating the graylevel intensities of the subsets. Put another way, if the subset moves by 0.2 pixels, some of the pixels will remain nearly the same gray-level intensity, but others near large gray-level gradients will not. Using an interpolation scheme allows the matching algorithm to resolve this 0.2 pixel movement. Also instead of being restricted to summing over gray-level intensities at exact pixel locations $\mathbf{x}_{i}$ in Eq. 1, one can now sum over a list of optimally placed sample points across a region of interest (ROI).

The reader may also notice that, as described thus far, minimizing a chosen $S$ will only produce accurate results for rigid body translation of the subset. Subset rotation and deformation are accounted for by introducing shape functions analogous to finite element shape functions. The most commonly used shape function assumes strains within the subset are uniform:

$$
\mathbf{x}_{i}^{*}=\mathbf{x}^{*}\left(\mathbf{x}_{i}, \mathbf{p}\right)=\left(\begin{array}{c}
p_{0} \\
p_{1}
\end{array}\right)+\left(\begin{array}{cc}
1+p_{2} & p_{3} \\
p_{4} & 1+p_{5}
\end{array}\right) \mathbf{x}_{i}
$$

where $\mathbf{p}$ is a vector of parameters $\left\{p_{0}, \ldots, p_{5}\right\}$. This allows us to write $S=S(\mathbf{p})$.

The optimal match between the reference subset and the current subset is found by minimizing $S$ with respect to $\mathbf{p}$. The Newton-Raphson method is currently the preferred means to determine $\mathbf{p}$ (although the recent Levenburg-Marquardt nonlinear parameter estimation scheme also shows promise ${ }^{17}$ ). Once $\mathbf{p}$ is found for all subsets, strains can be calculated as a post-processing step. The components of the displacement $\mathbf{u}=\mathbf{x}^{*}-\mathbf{x}$ are $(u, v)$, which correspond to $\left(p_{0}, p_{1}\right)$ for the shape functions of Eq. 4. Instead of using the displacement gradients $\left(\frac{\partial u}{\partial x}, \frac{\partial u}{\partial y}, \frac{\partial v}{\partial x}\right.$, $\left.\frac{\partial v}{\partial y}\right)$ that were calculated for each subset $\left(p_{2}, p_{3}, p_{4}, p_{5}\right)$, the displacement gradients are typically recalculated from the displacements $(u, v)$ at the center of each subset (a grid point). This way the displacement gradients at a point depend on the neighboring grid points, rather than relying entirely on the correlation analysis of one subset. From the gradients, Lagrangian strains (2-D here) can be determined by

$$
\begin{aligned}
& \varepsilon_{x x}=\frac{\partial u}{\partial x}+\frac{1}{2}\left[\left(\frac{\partial u}{\partial x}\right)^{2}+\left(\frac{\partial v}{\partial x}\right)^{2}\right], \\
& \varepsilon_{y y}=\frac{\partial v}{\partial y}+\frac{1}{2}\left[\left(\frac{\partial u}{\partial y}\right)^{2}+\left(\frac{\partial v}{\partial y}\right)^{2}\right], \\
& \varepsilon_{x y}=\frac{1}{2}\left[\frac{\partial u}{\partial y}+\frac{\partial v}{\partial x}+\frac{\partial u}{\partial x} \frac{\partial u}{\partial y}+\frac{\partial v}{\partial x} \frac{\partial v}{\partial y}\right] .
\end{aligned}
$$

Note that some degree of spatial smoothing is usually performed on the displacement gradients (and/or strains) to remove the noise amplified by taking derivatives of experimental data.

The 3-D DIC method utilizes two or more cameras that image the specimen from multiple viewpoints, in order to additionally capture the out-of-plane motions. 3-D DIC determines point correspondences between the two images acquired from the cameras at the same instant. To measure both the initial shape and the deformation of an object, the relative positions and operating characteristics of the two cameras must be determined through a calibration procedure using a series of images of a grid with a known pattern. Once the calibration parameters of each camera and their relative orientation in space are determined, the specimen shape can be reconstructed using triangulation. ${ }^{18}$

In general terms, the main parameters affecting the accuracy of the method are the following:

- Specimen surface specularity. Surfaces with random, fluctuating, gray-scale intensity distributions work best. Surfaces that have a matte color are much better than that those that are highly polished/reflective. Also, surfaces with monotone (uniform) or very gradually varying surface intensities may not work well. Consequently, unless the pre-existing surface happens to be satisfactory, one must often artificially apply a speckle pattern using paint or some other high contrast medium.

- Camera, lens, and environment. The usual rules for good digital photography apply here. One must pay attention to illumination, field of view (FOV), and depth of field (DOF) throughout an experiment. Also, it is usually best to perform DIC with direct line of sight to the specimen surface. Intervening 
mirrors or other optics that refract light introduce optical aberrations in the images, so we discourage their use unless the distortions can be corrected prior to correlation.

- Image spatial resolution versus speckle size. Image quality can be improved by using a high pixel resolution charge-coupled device (CCD) digital camera. Speckles should be large enough to encompass multiple pixels in an image (oversampled), but should not be so large that images cannot be correlated with the required spatial resolution. Saved images should be of a highquality, uncompressed format, say bitmap or tiff (not jpeg).

- Gray-scale dynamic range. A higher number of discrete gray levels (dynamic range) captured by the CCD camera and data acquisition system is generally better, but the usable dynamic range may be limited by illumination and the contrast of the surface pattern. A high bit A/D converter is advisable, and, if needed, the CCD signal can be amplified to utilize the full dynamic range of the A/D converter. Incidentally, color CCD cameras are not recommended, due to the way the CCDs are physically constructed, where the introduction of color introduces a non-monotonic intensity scale and reduces the effective dynamic range.

- Frame rate. The number of images taken must be sufficient to resolve the motion of the specimen. More images are better, provided one has sufficient storage capacity for them. One can always throw out images, if not needed during post-processing. If an insufficient number is taken, the relative displacements may be too large between sequential images, and the cross-correlation scheme may fail. This can usually be remedied by manually selecting an initial guess for the Newton-Raphson minimization of $S$ described above, but this may be inconvenient.

- Choice of interpolation function. As mentioned previously, gray-level intensity values and their derivatives between pixels are typically interpolated to reconstruct image intensity patterns prior to correlation. Higher order interpolation schemes increase the accuracy. A dramatic error reduction is achieved when going from a linear to a cubic interpolation, with rather diminishing improvements for fifth-order interpolation. ${ }^{19}$

- Out-of-plane displacements (2-D DIC only). 2-D DIC is imaged by one camera oriented perpendicular to the specimen surface and is best used for a planar surface under primarily in-plane deformation. In practice, however, out-of-plane displacements are difficult to avoid. The error induced by out-ofplane motion can be minimized by either increasing the distance between the camera and the object when using a standard lens, using a telecentric lens, or by estimating a functional form of the strain error from measurements of out-of-plane displacement at three points on the specimen. ${ }^{20}$ An especially poignant example where ignoring out-of-plane displacements can produce inaccurate measurements in the measurement of Young's modulus of steels is illustrated in Appendix A of Ref. 21.

- Field of view (3-D DIC only). The same material points should be in view of multiple cameras. This can be problematic for specimens with large curvatures, such as for imaging of thin wires of interest here. Out-of-plane measurement accuracy is improved by placing cameras at relatively large angles to each other, but small camera angles may be necessary to achieve a sufficient FOV overlap when imaging cylindrical objects.

When using 3-D DIC, the camera-lens system must be optimized to balance DOF and FOV for the specific test under consideration. An insufficient DOF can cause pattern defocusing due to out-ofplane motion and introduce substantial error in the calculated displacement fields. The specimen must stay in the cameras FOV and stay in focus for the entire testing period. Assuming a fixed lens size, a reduction in aperture size will improve the DOF but will require an increase in object illumination. A reduction in aperture size may also cause light to diffract through the aperture causing an out of focus image. An increase in the focal length of a lens will also increase the DOF but will decrease the angular FOV. ${ }^{5}$

Other sources of error can arise from poor pattern quality, poor specimen illumination, lens distortions, environmental disturbances, and complex testing environments (e.g., submerged specimens, high temperature testing). Sizeable error can also be introduced by the incorrect choice of speckle size and subset size. Sutton et al. ${ }^{5}$ recommend each speckle should fill a $3 \times 3$ pixel array in the image, as this gives near optimal spatial over-sampling. They also recommend that each subset contain at least $3 \times 3$ speckles to ensure reasonable matching accuracy. Typically linear shape functions are used (such as those of Eq. 4), so the selected subset must be small enough that the strain within the subset can be assumed constant yet large enough to encompass an area that has a statistically different pattern from the neighboring subsets. Computations show that 
if the signal contains a dominant frequency, the subset size should encompass at least half of this local wavelength in order to avoid misregistration (i.e., aliasing). ${ }^{22}$ Using a combination of speckle oversampling, accurate interpolation between pixels and image quantization with at least 8 bits can produce displacement fields with an accuracy of \pm 0.02 pixels or better as shown in Ref. 19.

More detailed, step-by-step, recommendations for assembling an experimental setup with DIC measurements are provided in section Experiment 2 Setup.

\section{Specimen Preparation}

The accuracy of the DIC measurements is especially dependent on the quality of the surface pattern, and since the pattern is usually applied artificially, specimen preparation is worth discussing in some detail. This takes some practice to obtain good results. The speckle pattern must be sufficiently non-periodic, isotropic, and of high contrast to guarantee the uniqueness of the tracking signature for each subset. Prior work has shown that a smooth transition between black and white within a given speckle is preferable (as opposed to uniformly black speckles on a white background, which fortunately in practice is actually difficult to achieve) for accurate measurements, and the pixel-to-speckle ratio must be sufficiently large to enable over-sampling. ${ }^{19,23}$ of course, the pattern application should not alter the mechanical or chemical characteristics of the sample. The pattern should have minimal local areas of reflection, so the use of nonreflective matte paints and diffuse lighting is recommended. If there are persistent reflective or otherwise corrupted pixels in the image, then the user should consider removing these points from the correlation analysis.

Optimal pattern application methods vary, depending on the required magnification. For microscale measurements, high-quality patterns can readily be obtained by airbrushing. Airbrushes can be purchased with different nozzles depending on the desired size of the pattern, where a smaller nozzle diameter produces smaller paint droplets (speckles). The pattern is often applied by lightly coating the sample surface with a light paint, letting this dry, then spraying the coated specimen with a dark mist of paint. Reversing the sequence, first dark paint, then light paint works equally well. (More detailed suggestions for airbrushing will be provided in section Specimen Preparation.) For materials where paint does not adhere well, such as certain polymeric materials, ink can be used to dye the specimen surface.

A problematic issue during dynamic or extreme temperature testing, particularly under large strains and when paint is used to apply the speckle pattern, is the tendency of the pattern to debond from the specimen surface. Recent experiments have shown that both the maximum surface strain and the strain rate are important factors when determining if a speckle pattern will debond from a specimen surface. $^{24}$ This can be mitigated by cleaning and lightly roughening the surface prior to painting, and by applying the paint immediately prior to the experiment.

Speckle pattern quality can be evaluated through gray-scale statistics, ${ }^{5,25}$ where an image intensity histogram is plotted with the gray-scale intensity of a pixel on the $x$-axis, and the number of pixels at that gray-scale value on the $y$-axis. A typical CCD stores 8 bits per sampled pixel, allowing 256 possible shades of gray to be recorded, so the $x$-axis of the histogram typically runs 0-255. A non-Gaussian (bimodal or otherwise skewed) image intensity profile can indicate a poor quality pattern, areas of local reflection, or other issues that can degrade correlation results. However, a Gaussian distribution alone does not indicate a high-quality pattern for DIC, as an overly smooth intensity could be Gaussian but not specular. Baseline translation and rotation testing is another indicator of pattern quality, where a known rigid body displacement is applied to the specimen and plotted against the DIC-measured displacements to assess the resulting quality of the measurement.

\section{Experimental Results}

Two experiments will be described below to demonstrate how DIC can be applied to experiments on SMA wire. The first experiment was performed at General Motors R\&D on Flexinol wire ${ }^{\circledR}$ subjected to Joule heating using a 2-D DIC setup. The second experiment was performed on SE wire at the University of Michigan using a 3-D DIC setup.

\section{Experiment 1: Joule heating response of SM wire}

In this first experiment, the deformation resulting from the martensite-to-austenite $\left(M^{+} \rightarrow A\right)$ temperature-induced phase transformation of SM wire was studied with 2-D DIC. In this simple setup, a prestrained SMA wire was heated above its transition temperature at constant load to induce contraction of the wire due to the shape memory 
(a)

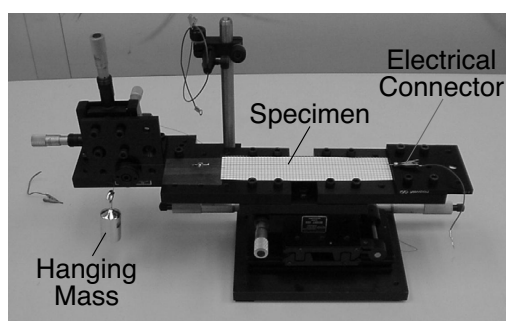

(b)

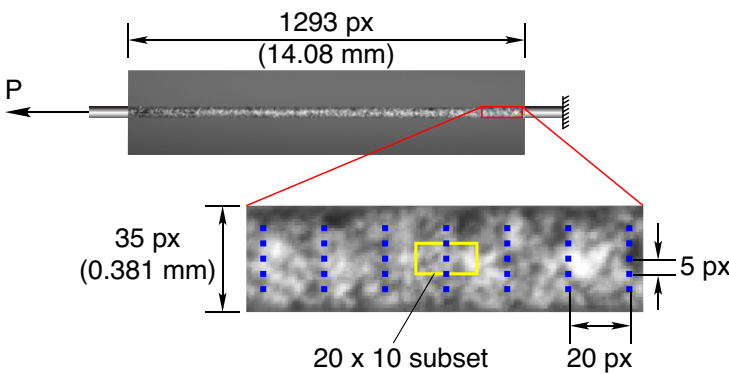

Figure 1 Experiment 1 - setup: (a) photograph of setup and (b) image and schematic of specimen with magnified image (inset) of speckle pattern.

effect. As-received $90^{\circ} \mathrm{C}$ Flexinol wire, $0.381 \mathrm{~mm}$ (0.015 inches) diameter (obtained from Dynalloy, Inc.), was chosen for this experiment. This material has a transformation temperature above room temperature $(\mathrm{RT})\left(A_{\mathrm{f}} \approx 80-90^{\circ} \mathrm{C}\right)$, is preconditioned by the supplier for actuator applications (with a max recommended load of $20 \mathrm{~N}$ ), and is provided to the user prestrained at RT. (We should also mention that this SMA wire has a significant two-way shape memory effect, ${ }^{26}$ so it will contract upon heating and lengthen upon subsequent cooling.) A wire specimen of approximate length $200 \mathrm{~mm}$ (8 inches) was placed horizontally on a mounting device with one crimped end fixed to a rigid mount (right end) and the other end attached to a $100 \mathrm{~g}(0.98 \mathrm{~N})$ deadweight via a rigid wire across a pulley as shown in Fig. 1(a). The axial stress level was small (8.6 MPa), just enough to keep the wire straight. The wire was suspended about $5 \mathrm{~mm}$ distance above the bottom surface of the mounting device to avoid any intervening contact between the ends. Also, the left end of the wire was allowed to displace significantly as the wire contracted or elongated, so new material could enter or leave the FOV. Care was taken to ensure that the central $60-70 \%$, or so, of the wire remained in the FOV of the camera during the entire experiment.

A gray contrast pattern was applied to each wire through separate treatments of white and black aerosol spray paint speckles. In this case, the wire was first coated with a fine dusting of white paint by holding the aerosol can about $0.3-0.45 \mathrm{~m}$ from the specimen and spraying for a few seconds. It was then sprayed with black paint in a similar manner to produce the speckle pattern shown in the photograph of Fig. 1(b). Alternatively, one could use white spray paint followed by a sprinkling of fine black laser toner powder, but the white and black paint worked well for our 0.381 -mm-diameter wires. The key point here is that speckles should be small enough that multiple speckles fit within the chosen pixel subset. Thus, one speckle should never exceed the dimensions of the chosen pixel subset. A grid point is the center point of a subset where the vector $\mathbf{p}$ is calculated. Keep in mind that uniqueness from grid point to grid point in the DIC process is only guaranteed (within a certain numerical tolerance) by the subset of pixels surrounding each grid point. In other words, the purpose of the pixel subset is to help the software find each grid point from image to image.

During the experiment, the temperature of the wire specimen was increased by Joule heating from RT to above the Flexinol transformation temperature. A power supply was connected to both ends of the wire, and the electric current was increased from 0 to $1 \mathrm{~A}$ at approximately $0.01 \mathrm{~A} / \mathrm{s}$. The current (1 A) was then held constant throughout the remainder of the experiment until no more deformation was observed, indicating that $M^{+} \rightarrow A$ phase transformation had ended.

Imaging was performed from above by a single CCD camera (Sony XCD-SX900) with a $1280 \times 960$ pixel array and a maximum frame rate of 7 frames/s. A telecentric lens affixed to the camera captured images of a central portion of the wire. The DASYLab software package ${ }^{27}$ was used to control the camera and to record the axial load and displacement data. Images were taken at about 2-s intervals (total of 46 images), and the axial load and displacement were recorded at a sampling rate of $3 \mathrm{~Hz}$.

After the images were acquired (in bitmap BMP format), the SDMAP software (Surface Deformation Mapping Program) developed at Yale University ${ }^{28,29}$ was used to compute the strain fields. The approach to strain mapping is similar to that of Refs. 30-32. The six parameter affine deformation mapping function of Eq. 4 (constant displacement gradients) was employed for the deformation of each $20 \times 10$ subset. A bicubic spline interpolation was used for subpixel gray-scale values. The correlation algorithm of Ref. 30 was used, although others are available. This was found to be faster than that in Ref. 32 with comparable performance. 
The DIC analysis computed the strains from the finite deformation formulation, Eq. 5, in terms of the displacement gradients. These were obtained through a post-DIC processing routine (a moving least square filtering routine) of the grid displacements. Small, rectangular pixel subsets $(20 \times 10)$ were employed, instead of the usual square subsets, due to the narrow geometry of the wires. Although the primary interest was the axial strain, a narrow subset permitted 5 grid points, spaced 5 pixels apart, to be analyzed in the transverse direction. The images in the present study were compared in an incremental fashion, in which the current (deformed) image was compared against the previous (reference) image to give the increment in the displacement and displacement gradient fields. These were then added to obtain the displacement and displacement gradient fields relative to the first reference image.

In 2-D DIC of a wire, the cylindrical surface is projected onto the planar CCD of the single camera, so only the crown of the wire is not significantly distorted by curvature effects. The measurements of $\varepsilon_{x y}$ and $\varepsilon_{y y}$ would not be expected to be accurate near the edges of the wire. Measurements of $\varepsilon_{x x}$ would be less affected by curvature, but some error may still exist at the edge as the Lagrangian strain $\varepsilon_{x x}$ in Eq. 5 would include an inaccurate $\partial v / \partial x$ as a second-order term. Thus, to be conservative, displacement and strain fields were computed over a ROI of $1181 \times 21$ pixels centered along the wire crown, rather than across the entire 35 pixel wire diameter. This corresponded to an analysis grid of $60 \times 5$ with $20 \times 5$ pixel spacing, as shown in Fig. 1 (b).

Initially, the reference image was taken at RT (as was convenient), so the relative axial strains recorded during the experiment were negative. We performed one last post-processing step by subtracting the axial strain field of the last image (highest temperature) to make Austenite the reference configuration, as is conventional. Figure 2 shows the average strain over the entire ROI $\left(\bar{\varepsilon}_{x x}\right)$ for each image taken. It shows how the $4.26 \%$ prestrain in the martensite (at RT) is recovered during heating, decreasing gradually, then steeply, then gradually again, reaching zero by the last image. Some points in the figure (solid circles) are tagged at selected times $\left(t_{1}, \ldots, t_{11}\right)$ to show details in subsequent figures.

Figure 3 shows the evolution of axial strain field, $\varepsilon_{x x}(x, y)$, along the wire crown for the 11 selected times of Fig. 2. Overall, the strain profiles translate downward in the plot as time (and heating) progress (similar to the trends in Fig. 2), but it also shows some interesting and unexpected features. The axial strain

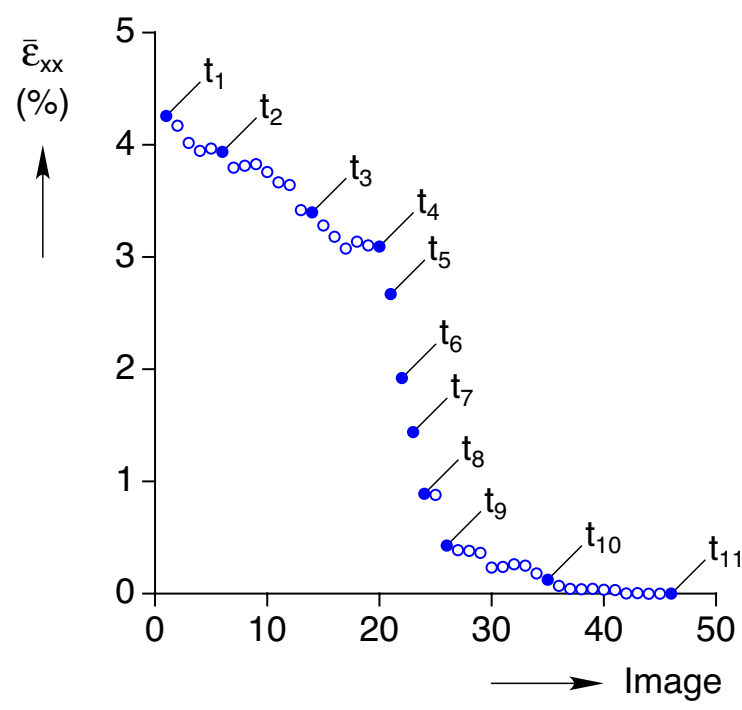

Figure 2 Experiment 1 -average axial strain $\left(\bar{\varepsilon}_{x x}\right)$ evolution of Flexinol during Joule heating

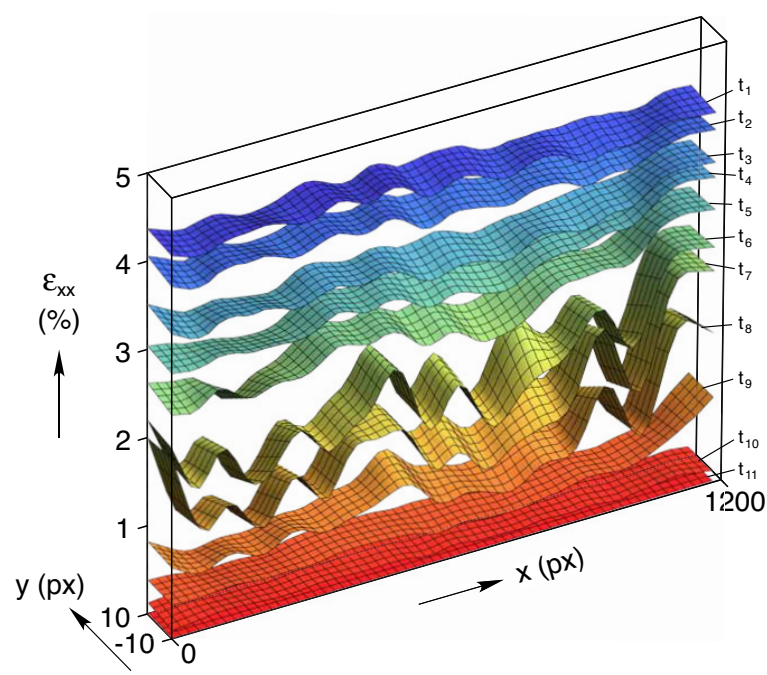

Figure 3 Experiment 1 -axial strain contours at selected times.

is essentially constant across the DIC ROI at $t_{1}$ (with some minor waviness). Between times $t_{6}$ and $t_{8}$ the axial strain became quite wavy ( $\pm 0.5 \%$ strain), especially in the axial direction, and then became flat again by time $t_{10}$. The cause of the waviness is not known with certainty, as we do not know the exact preconditioning procedure used for Flexinol wire (which is proprietary), but our measurements indicate that small pockets of material undergo transformation at different times. We suspect that residual stresses, creating by the preconditioning, create local heterogeneities in the "driving force" which influence local nucleation sites for early (or late) transformation. 


\section{Experiment 2: Cyclic shakedown of SE wire}

We now turn to the second experiment to demonstrate the cyclic shakedown behavior of SE wire (same alloy as used in previous parts of this series) at RT, using 3-D DIC measurements to quantify changes in recovery strains, along with simultaneous infrared (IR) imaging to quantify changes in latent heat and thermo-mechanical coupling.

Cyclic shakedown of Nitinol is an important aspect that SMA application engineers must often face for adaptive structures applications, as repeatable cyclic behavior is essential for the success of many devices. Usually, however, as-received NiTi wire exhibits significant changes in its response, including transformation stresses, recovery strains, and latent heat changes, when subjected to thermo-mechanical cycling. This can create difficulties for application engineers who wish to design devices, both for thermally induced actuation (like the previous experiment) and isothermal shape recovery (SE). To cope with this, SMA wire is often "trained" prior to installation in the device to achieve a desired response through repetitive, carefully controlled thermomechanical cycles that are representative of expected operating conditions. The result is "shakedown" (or cyclic stabilization) to the desired material response (see, e.g., Refs. 26, 33, 34).

\section{Specimen preparation}

To have an independent (and confirmatory) measurement of the strain during the experiment and to control the strain amplitude during cycling, reflective tags were attached to the specimen to use a laser extensometer (LE). To get a reliable reading, the width of the laser tags must extend beyond the diameter of the specimen $(d=0.762 \mathrm{~mm})$, so tabs were made of epoxy on the back side of the specimen to affix the LE tags (see left-hand image in Fig. 4). Care was taken to leave the front of the wire free of epoxy to permit DIC measurements. After the epoxy had cured, the specimen was first painted with a background coat of Golden Airbrush Titanium White (\#8380), followed by a speckle coat of Golden Airbrush Carbon Black (\#8040). These paints were chosen for their opacity, strong contrast, and good adhesion even under superelastic strains. They were both applied using an Iwata custom micron-B airbrush, which has the smallest nozzle in its class $(0.18 \mathrm{~mm})$. Through experimentation it was found that the smallest speckles were produced by pulling the airbrush needle back as little as possible to allow paint to flow, and setting the air pressure as high as

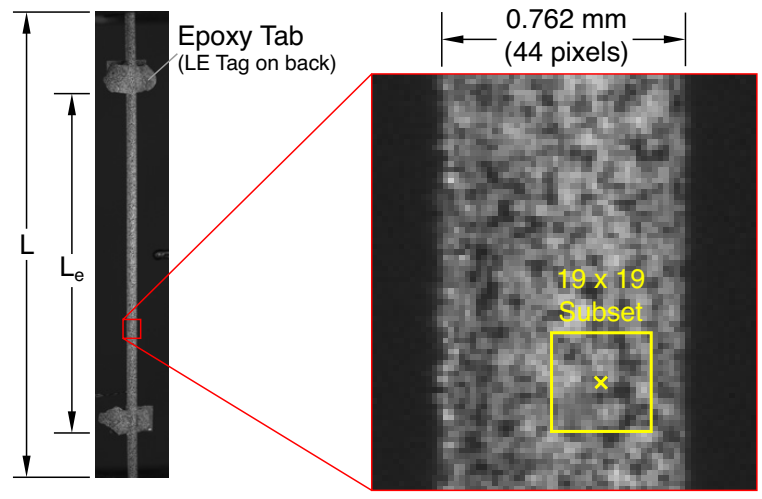

Figure 4 Superelastic specimen and speckle pattern.

possible. This combination of a small opening for the paint and high pressure (340 $\mathrm{kPa}$ in our case) caused the paint to atomize upon exiting the airbrush. The airbrush was held about $50 \mathrm{~mm}$ away from the specimen during paint application. To quickly assess the resulting speckle pattern, we then took an image of the specimen using the cameras and field of view of our setup, and confirmed that speckles were approximately $3 \times 3$ pixels in size as recommended by Sutton et al. ${ }^{5}$ A magnified view of the resulting image is shown as the inset of Fig. 4, showing 44 pixels across the wire diameter and a typical $19 \times 19$ subset used in the correlation analysis.

\section{Experiment 2 setup}

The experimental setup was similar to that described in our previous articles for a RT superelastic experiment, but with the addition of optical imaging for DIC. The front view of a typical imaging arrangement of a vertically oriented specimen is shown in Fig. 5. An IR camera (Inframetrics ThermCam SC1000 IR imaging system with a $256 \times 256$ pixel array) measured the specimen's temperature field. The emissivity of the painted specimen was measured to be $\epsilon=0.91$, which is close to the ideal emissivity of 1 . The two optical cameras shown on either side of the IR camera are Pt. Grey Research Grasshoppers (GRAS-50S5M-C). Each is a rather compact camera with a $2448 \times 2048$ (3.45 $\mu \mathrm{m}$ square pixels) black and white CCD. The manufacturer reports the Grasshopper is capable of 14 bit gray-scale resolution, but the effective (noise free) dynamic range was about 8 bits for our setup. We should mention that single lens reflex (SLR) digital cameras are not ideal for DIC because the quick movement of the mechanical mirror shutter system causes the camera body to shake slightly, as opposed to the electronic frame shutter utilized in 
the Grasshopper. Tamron CCTV 75-mm focal length lenses (as shown in Fig. 5) have been successfully used in previous experiments, but here we used 75-mm Fujinon HF75SA lenses instead (not shown) which had a maximum aperture diameter of $41.6 \mathrm{~mm}$ (vs. $19.2 \mathrm{~mm}$ for the Tamron lenses). This meant it was possible to rely on the diffuse fluorescent lights described below and use very little direct lighting, which can cause glare.

The specimen was clamped between hardened steel plates within pneumatically actuated grips resulting in an axial ( $x$-direction) free length of $L=36.96 \mathrm{~mm}$. It was loaded uniaxially in displacement control by an Instron 5585 electro-mechanical, lead-screw driven, load frame, where the lower grip was fixed and the upper grip displacement $(\delta)$ was controlled and measured by the load frame's high resolution optical encoder. A $500 \mathrm{~N}$ load cell monitored the resultant axial force $(P)$. From the back side, an Electronic Instruments Research (model LE-05) LE measured the elongation $\left(\delta_{\mathrm{e}}\right)$ between the laser tags affixed to a central gage section of initial length $L_{\mathrm{e}}=28.57 \mathrm{~mm}$. The upper grip displacement $\dot{\delta}=\mathrm{d} \delta / \mathrm{d} t$ was prescribed at a constant rate during loading $(\dot{\delta}>0)$ and unloading $(\dot{\delta}<0)$. As we have mentioned before in previous parts, some disagreement between local and global strain measurements is typically unavoidable due to some grip slippage as transformation fronts (localized strains) enter or exit the free length. Artifacts from grip slippage were (mostly) eliminated by reporting

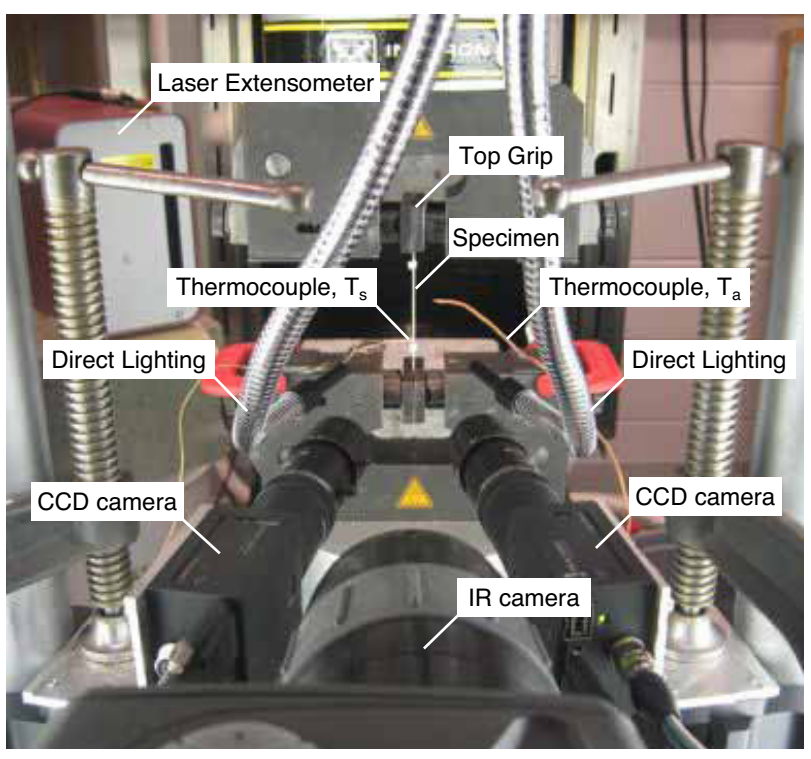

(a) the strains from the $\operatorname{LE}\left(\delta_{\mathrm{e}} / L_{\mathrm{e}}\right)$ or DIC $(\varepsilon)$ rather than the global strain $(\delta / L)$. The prescribed "global strain rate" was $\dot{\delta} / L= \pm 5 \times 10^{-4} \mathrm{~s}^{-1}$, which should be emphasized again, is quite different than the "local strain rate" when transformation fronts are propagating.

In setting up the cameras, many of the same techniques known to any photographer were employed. Here is the procedure we used:

1. Clean the lens and protective glass in front of the CCD. If a certain area of a speckle pattern will not correlate, and there does not appear to be anything wrong with the focus, lighting, or the speckle pattern, then the problem may be dust on the lens or protective glass in front of the CCD. However, to correct this one must touch the cameras and destroy the calibration. So, prior to camera placement in the setup, we held white paper in front of the camera, outside of the focal range, and slowly moved it around. The live camera images will appear as pure white if the lens and CCD protective glass are clean. If, however, gray smudges exist that do not move as the paper is moved, then the lens should be cleaned. If the smudges remain and they do not move when the lens is rotated inside its C-mount, then the source of the smudges is on the protective glass of the CCD, and blowing purified compressed air across the protective glass should correct the problem.

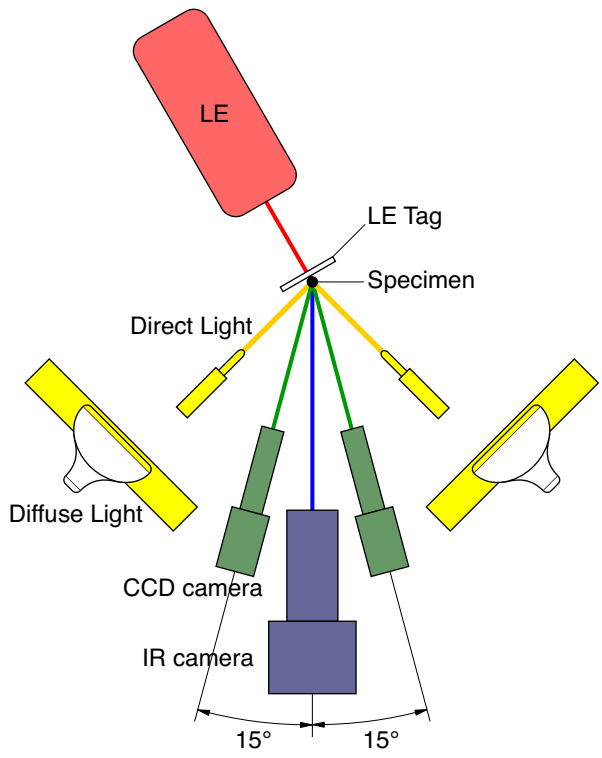

(b)

Figure 5 Superelastic experiment setup: (a) photograph of front view and (b) schematic of top view. 
2. Place and orient the cameras. The cameras were placed on an optical table in front of the testing frame. In this setup, the bottom grip of our setup was fixed and the top grip moved with the crosshead, so the bottom of the FOV was aligned with the bottom of the specimen and the top of the FOV included $13 \%$ above the top of the specimen to allow for specimen elongation. The total FOV height was $45 \mathrm{~mm}$, and the inclusive angle between the cameras (stereo angle) was $31.4^{\circ}$. (In past experiments, as shown in Fig. 5, we have used $18-20^{\circ}$, a somewhat better FOV overlap between the cameras, but in this case the IR camera interfered.) At this point, the cameras were rigidly clamped down, and the data/power cables were tied down to the camera mounting posts.

3. Focus the cameras and set the aperture. To center the DOF across the specimen, the aperture was initially fully opened to $f / 1.8$ (41.67 $\mathrm{mm}$ diameter). This reduced the DOF to its minimum, making it easy to set the working distance and focus the camera on the specimen. The aperture was then closed back down to increase the DOF. Although the aperture could be closed down to $f / 22$ (3.4 mm) to attain the maximum DOF, the image became noticeably blurry after $f / 8(9.4 \mathrm{~mm})$. Owing to the especially small pixels of these cameras $(3.45 \mu \mathrm{m})$, Airy circles from light diffracting through the small aperture were larger than a pixel, causing the blurriness. Close side-by-side comparison revealed that even an aperture of $f / 8$ produced a slightly blurred image compared to $f / 5.6$ (13.4 mm), so the aperture was set at $f / 5.6$. From this point on, the cameras were not disturbed and equipment around the cameras was only touched carefully when necessary.

4. Calibrate the camera orientations and positions. A 3-D DIC analysis requires the precise orientation of the cameras relative to one another. This is typically performed by taking a number of images with both cameras of a known calibration grid in different positions and orientations. Only 5-10 images are required, but more are better to reduce the measurement uncertainty and quantify the effect of lens distortions. To calibrate the cameras without disturbing them, the specimen was removed from the grips, and the calibration grid shown in Fig. 6 was placed in view of the cameras. Having closed down the aperture meant less light was reaching the CCD, so the lighting and exposure time were adjusted to compensate. The calibration grid was attached to a three-axis gimble and two micrometer stages, which were held in the top grip.

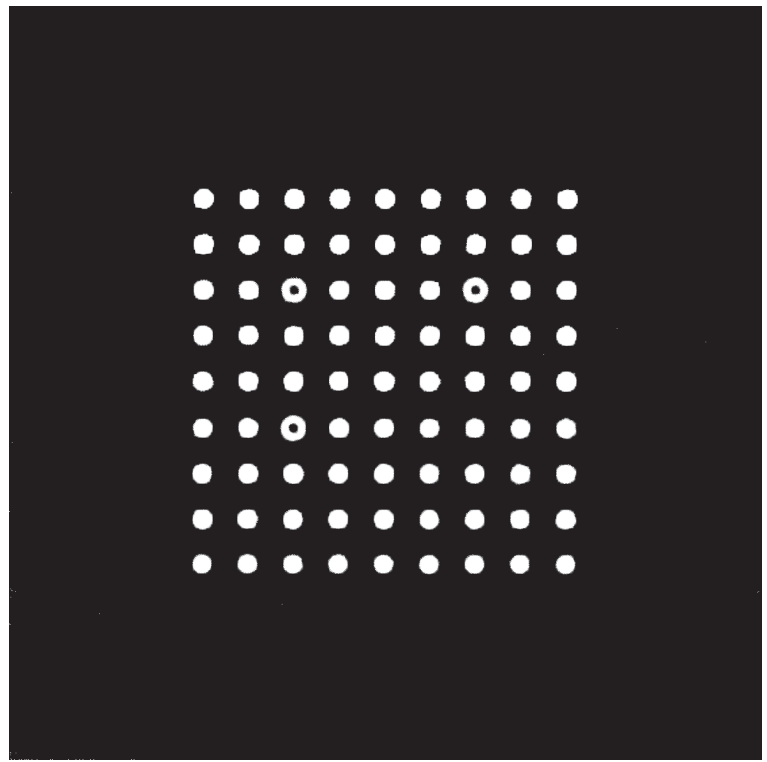

Figure 6 Vic-3D calibration grid.

Along with the movement of the crosshead, all six degrees of freedom were available to translate and rotate (approximately $\pm 20^{\circ}$ ) the calibration grid to generate 40 different pairs of images of the calibration grid within various locations of the FOV.

5. Set the lighting and exposure time. With the wellknown temperature sensitivity of NiTi, special care must be taken to choose lighting sources that do not change the temperature of the specimen. Here, we used fluorescent lights behind frosted translucent plastic (just out of view in Fig. 5(a)), similar to that of a light box, to produce a diffuse, flat, light. In addition, flexible fiber optic lights (coming in from the top of Fig. 5) with an adjustable light intensity were used to fine tune the illumination. The LE shown in Fig. 5 was placed behind the specimen to avoid shining the laser sheet on the same side of the specimen as the optical cameras. In addition, care was taken to not shine the fiber optic lights directly at the LE, as that would affect the extensometer reading. The camera exposure time was set at $20 \mathrm{~ms}$ using the camera control software, Vic-Snap. ${ }^{35}$ Given sufficient light, it is usually better to minimize the motion blur by selecting shorter exposure times. However, we have found exposure times less than $1 \mathrm{~ms}$ with the Grasshopper cameras produce noisy images. We believe that longer exposure times "integrate out" the noise in the light reaching the CCD sensor. Given this balance between motion blur and noise, our shutter time corresponds to 0.1 pixels or less of movement during the exposure. Finally, when 
selecting an exposure time/lighting combination, saturated pixels should be strictly avoided, as DIC performs its matching between the reference and deformed subsets using gray-level gradients. If a collection of neighboring pixels are completely white, there is no gradient, and usually will cause the correlation routine to fail at that location.

6. Perform a trial run. Before running any experiment it is advisable to take a few images of the specimen while it is static, load these images into the DIC software, and run a quick correlation. This can help locate problem areas due to lighting glare, inadequate speckle pattern, etc. It can also give you a dry run, to test out your camera triggering system. Here, the cameras were triggered with a function generator using a $0.8 \mathrm{~s}$ period $0-5 \mathrm{~V}$ square wave. This same function generator signal was also sent to the data acquisition system that captured the load, LE, thermocouples, and crosshead displacement data to synchronize all measurements.

After the experiment, the DIC images were analyzed using the commercially available Vic-3D software $^{36}$ across an ROI, centered on the crown of the wire $(2114 \times 16$ pixels $)$. The $3 \times 3$ rule $(3 \times 3$ pixels per speckle with $3 \times 3$ speckles per subset) is a good guide to quickly assess speckle size, speckle spacing, and pixel subset size. In general, larger subsets lead to better correlation between the reference image and the deformed image, but subsets that are too large can result in "over-smoothing" of the strain fields. Vic-3D uses the same constant strain shape functions by Eq. 4 as was used in Experiment 1. Thus, the choice of subset size is a trade-off between the correlation strength and the desired spatial resolution of strain inhomogeneities to be resolved. Our $19 \times 19$ subset included roughly $3 \times 3$ speckles and corresponded to a dimensional region of about $0.33 \mathrm{~mm} \times 0.33 \mathrm{~mm}$ (or $0.43 d \times 0.43 d$ in wire diameters). The analysis grid chosen was $1057 \times 8$ with $2 \times 2$ pixel spacing. We used Vic-3D's default cross-correlation function, the normalized sum of squared differences, Eq. 3. In contrast to SDMAP in Experiment 1, where the increment in strain was calculated by comparing the current deformed image against the previous deformed image, Vic-3D calculates the (total) Lagrangian strain by comparing the current deformed image against the reference (zero strain) image. The incremental method produces a better correlation between images, as for a smaller deformation increment the constant strain in the subset is a better assumption. However, the incremental method can lead to measurement error accumulation as more and more images are compared. On occasions when automatic correlation fails in Vic-3D (such as when material leaves the FOV then returns), the software allows the user to manually choose a "seed point" in the current image to help the software find a correlation match.

\section{Experiment 2 results}

In this experiment, the wire was cycled 25 times between $\delta_{\mathrm{e}} / L_{\mathrm{e}}=7.5 \%$ and $P / A_{0}=6.6 \mathrm{MPa}$ (a small load, just to keep the wire taut) in RT air $\left(22^{\circ} \mathrm{C}\right)$ at a global strain rate of $\dot{\delta} / L= \pm 5 \times 10^{-4} \mathrm{~s}^{-1}$. The mechanical response shown in Fig. 7 exhibits typical superelastic shakedown behavior, where the upper plateau evolves downward in stress and the residual strain at near zero load ratchets forward in progressively smaller increments with cycling. In addition, the length of both plateaus decreases, the stress-strain response becomes more sigmoid-like in shape, and the magnitude of the hysteresis (area within the loop) diminishes. All these features are well-known aspects of uniaxial NiTi wire behavior (see Ref. 33 for more details).

IR images and DIC images were taken at 1.25 frames/s during the experiment. Figure 8 shows an IR and a DIC snapshot at $t=121 \mathrm{~s}$, corresponding to $\delta_{\mathrm{e}} / L_{\mathrm{e}}=2.75 \%$ during the first-cycle upper plateau in Fig. 7. It shows a single $A \rightarrow M^{+}$front propagating downward that is detected in the IR image as a local

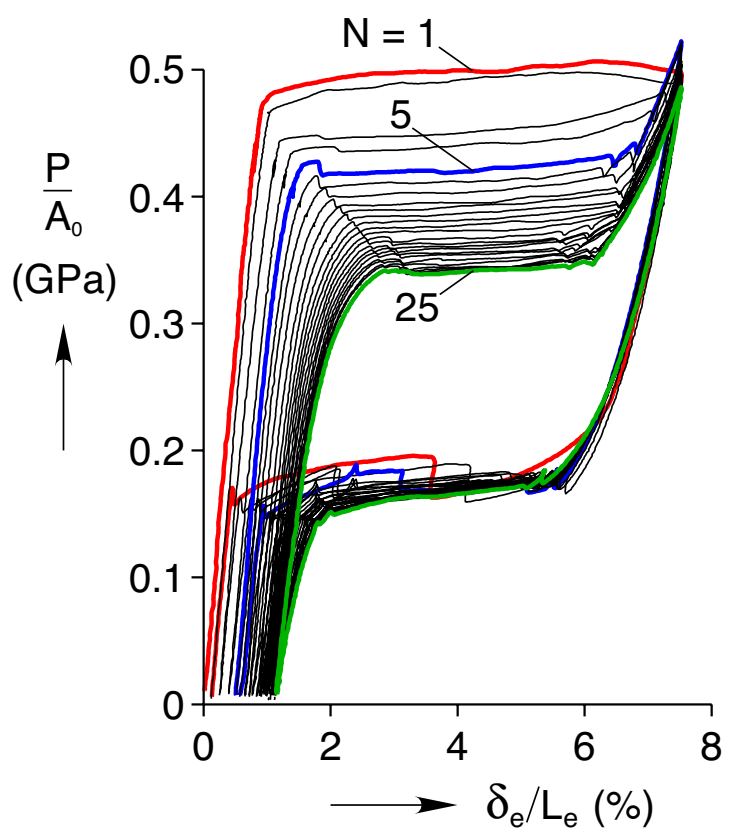

Figure 7 Experiment 2, superelastic mechanical response to 25 loadunload cycles in room temperature air $\left(22^{\circ} \mathrm{C}\right)$. 


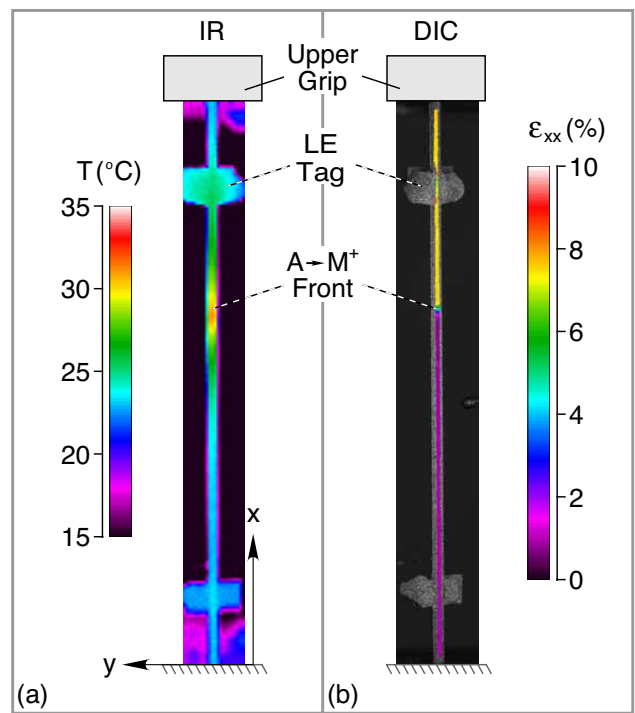

Figure 8 Experiment 2 , snapshots at $t=121 \mathrm{~s}$ of temperature (a) and axial strain (b) fields during first-cycle loading ( $A \rightarrow M^{+}$propagation).

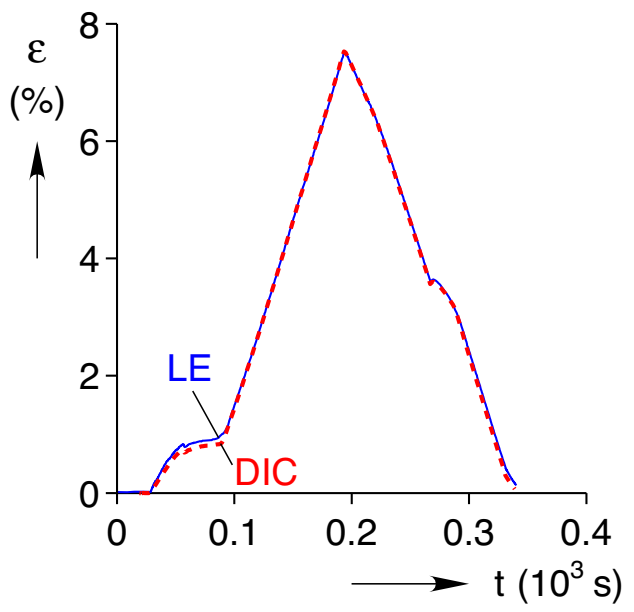

Figure 9 Experiment 2, cycle 1 comparison of average strains as measured by the laser extensometer (LE) and DIC.

hot spot and in the DIC image as a near discontinuity in axial strain from 0.9 to $7.5 \%$ (about 2 wire diameters in axial extent). As a confirmatory step, we compared the average axial strains between the LE tags as measured by the LE and DIC, which track together nicely (Fig. 9).

Figure 10 shows contour plots of the axial strain field history, $\varepsilon_{x x}(x, t)$, and temperature field history, $T(x, t)$, during the first five cycles. In each plot the wire axial coordinate $(x)$ is normalized by the initial free length $(L)$, as shown on the left-hand vertical scale. The stress history is overlaid for reference, and its scale is the right-hand vertical axis. The axial strain contour plot was created by averaging the strain across the lateral $(y)$ direction of the wire to create a l-D axial profile from each DIC image. A selected sequence at 1.6-s intervals was then stacked side by side $(\approx 200$ images per cycle) and synchronized with time $(t)$ along the horizontal axis. (3-D DIC provides a wealth of information, but here we are mostly interested in axial strains. Other plots of other strain components, such as lateral $\left(\varepsilon_{y y}\right)$ and shear $\left(\varepsilon_{x y}\right)$ strains, have also been processed that show similar discontinuities where fronts exists, but are not shown here in the interest of space.) The temperature contour plot was similarly created from the IR images at 1.6-s intervals $(\approx 200$ images per cycle). Both fields are shown in the Eulerian frame (current configuration), so the top of the free length moves up during loading and down during unloading according to the upper grip displacement.

During the first cycle, the sharp transition between high strain and low strain regions in Fig. 10(a) clearly shows transformation fronts (locations of high strain gradient) traversing the specimen length. As discussed in Part 4, these fronts also manifest themselves as local temperature changes in the specimen, which can be seen in Fig. 10(b), due to the exothermic ( $A \rightarrow M^{+}$during loading) and endothermic $\left(M^{+} \rightarrow A\right.$ during unloading) latent heat (enthalpy) exchanges with the environment. Because stagnant air is a relatively poor heat transfer medium, $A \rightarrow M^{+}$transformation creates self-heating and fronts show up as local hot spots (above RT); whereas, $M^{+} \rightarrow A$ transformation creates self-cooling and fronts show up as local cold spots (subambient temperatures). We have used this to our advantage in past work to track the transformation kinetics in SMAs. At this relatively slow loading rate, a single $A \rightarrow M^{+}$transformation front starts at the top grip where there is a stress concentration due to clamping, and it propagates downward at nearly constant speed until $x / L=0.1$ when the crosshead motion is reversed. The $A \rightarrow M^{+}$strain jump is about $\Delta \varepsilon_{x x}=7.5-0.9=6.6 \%$. During unloading, this initial front remains static momentarily until the stress reaches the lower plateau, at which point it reverses direction, propagating upward until a new $M^{+} \rightarrow A$ front starts from the top grip. These two fronts then propagate toward each other at about half the initial speed until they coalesce (annihilate each other) at $x / L=0.67$, at which point the end of the lower stress plateau is reached and further unloading occurs via a uniform strain field. The $M^{+} \rightarrow A$ strain jump is about $\Delta \varepsilon_{x x}=0.3-6.0=-5.7 \%$. 

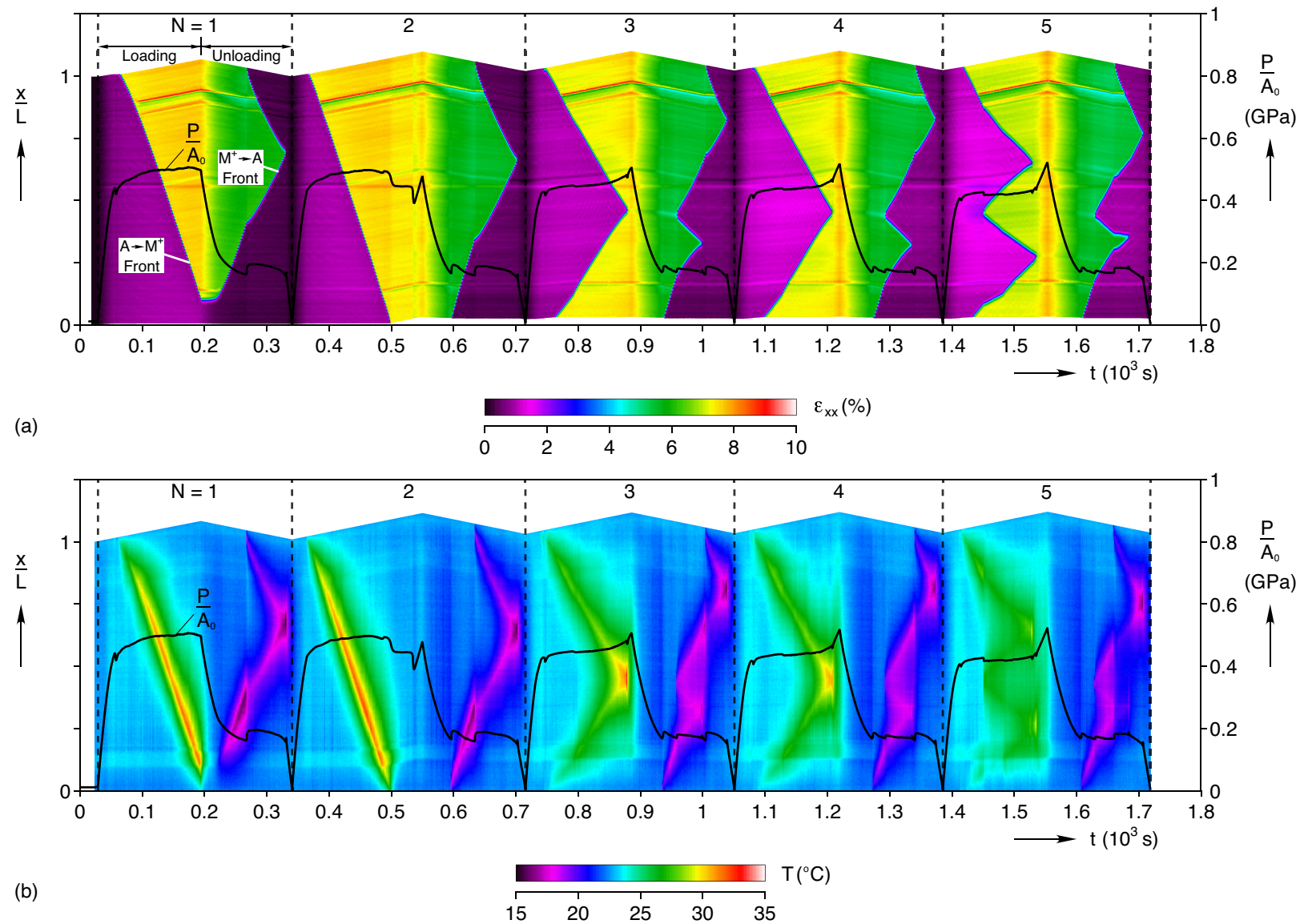

Figure 10 Experiment 2, cycles 1-5: (a) axial strain field history, (b) temperature field history.

The magnitude of the slope of each strain discontinuity (front) in Fig. 10(a) is related to its front speed $(c)$. Recall from Part 4 that transformation fronts "prefer" to propagate at the same speed according to

$$
c=\frac{\dot{\delta}}{n \Delta \varepsilon_{\mathrm{P}}}
$$

where $\dot{\delta}$ is the elongation rate, $n$ is the number of front propagating, and $\Delta \varepsilon_{P}$ is the strain jump across the stress plateau. This is really just a statement of axial compatibility between the grips, written in rate form, for steady-state (equilibrium) conditions. As new fronts are generated (increasing $n$ ) they can slow down, since mechanical equilibrium requires the axial force to be constant along the length and they each contribute to the prescribed the prescribed global elongation rate $(\dot{\delta})$. On the other hand, if the strain jump $\left(\Delta \varepsilon_{P}\right)$ decreases, as it does from cycle to cycle, they speed up. In the absence of boundary effects or other inhomogeneities that would change the local temperature or stress conditions, all fronts tend to propagate at the same rate, as this minimizes the local self-heating (or self-cooling) in the specimen and is energetically favorable.

The axial strain field is useful to precisely locate fronts (compared to the temperature field that is more diffuse due to axial heat conduction) and to quantify strain jumps, but the temperature field is useful to explain the stress history, as was shown in Part 4. The thermo-mechanical coupling in NiTi is clearly illustrated in Fig. 11, where the stress closely follows the trends of the temperatures $T_{A \rightarrow M^{+}}$and $T_{M^{+} \rightarrow A}$, consistent with the ClausiusClapeyron relation, Eq. 1 in Ref. 4, for transformation stress versus temperature. These transformation front temperatures were simply found from the maximum and minimum temperatures in the gage section during front propagation.

Returning to Fig. 10(a), one may notice three anomalies in the strain field history that exist during all cycles. Two of the anomalies are real disturbances in the strain field caused by the epoxy tabs for the laser tags at $x / L=0.13$ and 0.86 . The DIC strain measurements capture the fluctuations 


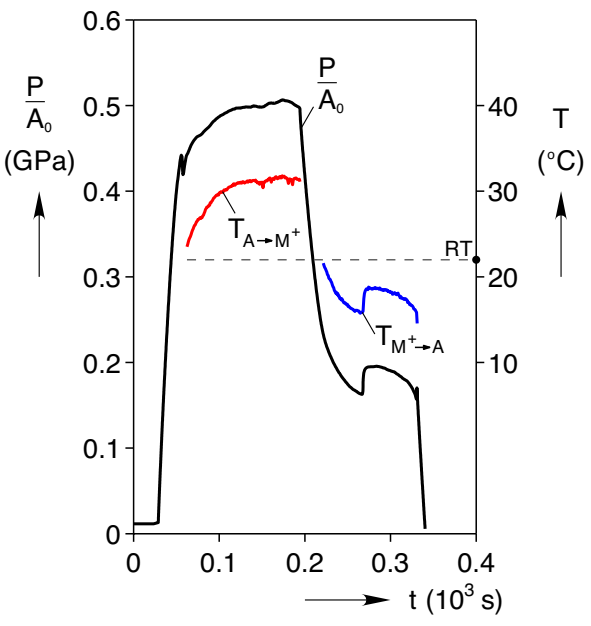

Figure 11 Experiment 2, cycle 1 stress and front temperature histories.

in strain caused by the constraint imposed by the epoxy mass and the slight stress concentration at these locations. The third anomaly consists of two slight horizontal streaks (discontinuities) at about $x / L=0.56$. The bottom streak does not follow the specimen displacement, while the upper streak does, which indicates a flaw in the CCD that affected both the deformed images and the reference image. This was confirmed by changing lenses and observing the same artifact on a new specimen. The bottom streak was even in the exact same pixel location. Thus, the streaks are an artifact of the CCD imperfection and should be disregarded.

The transformation fronts in the second cycle propagate along similar paths as in the first cycle. The only significant difference is that the $A \rightarrow M^{+}$ front reaches the bottom of the gage length, where, as frequently happens, the lateral contraction at the front caused the wire to slip out of the bottom grip a small amount. This slippage can be seen as a drop in the stress history as the front reaches the lower grip. It also created a rigid body translation of the material points between $t=500 \mathrm{~s}$ and $t=535 \mathrm{~s}$. Because sudden stress drops do occur for other (valid) reasons in SMA wire, it can often be difficult to determine whether load discontinuities are due to phase transformation or experimental problems. Here, DIC clearly identified this as an experimental problem, not the actual material response. The transformation did not reach the bottom grip during the first cycle but did during the second cycle. The reason was that the load frame was programmed to reverse the crosshead direction when $\delta_{\mathrm{e}} / L_{\mathrm{e}}>7.5 \%$ (not the global strain). The average strain in the $M^{+}$region reached $\varepsilon_{\text {avg }}=7.53 \%$ (by DIC) in the first cycle, but only $\varepsilon_{\text {avg }}=7.45 \%$ (by DIC) in the second cycle due to superelastic shakedown, so the crosshead proceeded further during this cycle before $\delta_{\mathrm{e}} / L_{\mathrm{e}}>7.5 \%$.

The remainder of the cycles exhibited typical superelastic shakedown (and no further significant grip slippage occurred). As the cycle number $(N)$ increases, we see an increasing number of fronts (Fig. 10(a)), probably due to accumulated defects in the wire that reduce the energy barriers for strain localization. One can see that the strains in the "austenite" (low strain) regions evolve to slightly increased strains, consistent with the small amount of residual strain accumulation (see again Fig. 7), which results in a decrease in strain jump across the fronts. With cycling the upper plateau moves downward in stress and its length (in time, or equivalently, $\Delta \varepsilon_{\mathrm{P}}$ ) decreases. This means that transformation via front propagation is exhausted at earlier global strains, consistent with the somewhat higher front speeds according to Eq. 6. Further transformation involves a nearly uniform strain field, and the stress rises steeply to its (nearly) same value at maximum elongation. This is the reason for the progressively larger stress "spikes" seen at the end of each loading increment.

Propagating fronts during later cycles are also accompanied by progressively less temperature deviations from RT than the first cycle, as shown in Fig. 10(b). The stress history exhibits a number of small jumps and drops, each of which (except for the grip slippage problem at $500 \mathrm{~s}$ in cycle 2) can be associated (and explained) by a change in front kinetics, in which fronts are created, annihilated, or change speed. By cycle 5 there are as many as four $A \rightarrow M^{+}$fronts (during loading) and three $M^{+} \rightarrow A$ fronts (during unloading), and some fronts even start and stop (see the unloading portion of cycle 5), apparently due to inhomogeneous defect accumulation in the wire that affects the local thermodynamic driving force for front propagation.

By cycle 25, as shown in Fig. 12(a), there exist as many as eight fronts during loading and four fronts during unloading, with some again propagating in "fits and starts." The fronts in the IR images (Fig. 12(b)) are now quite muted, although still discernible upon close inspection. This occurs for two reasons: (1) the smaller strain jumps and residual strain accumulation indicate that local plasticity has locked-in micro pockets of residual martensite, such that a smaller fraction of the material (within a given wire cross-section) actually participates in the transformation to release or absorb latent heat, and (2) more fronts during the transformation effectively distribute the self-heating and self-cooling more evenly 

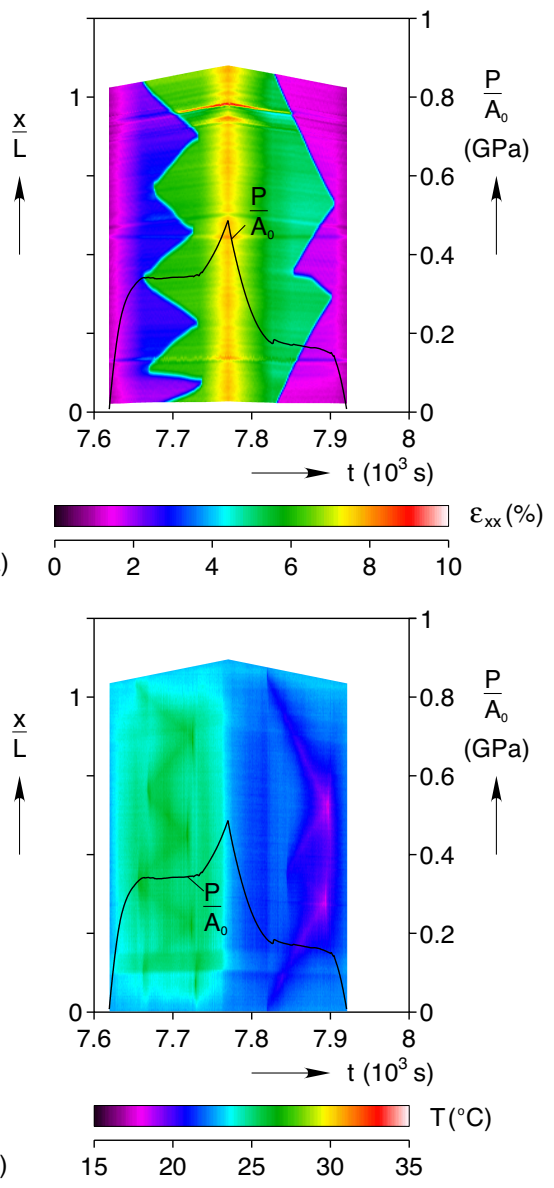

Figure 12 Experiment 2, cycle 25: (a) strain field history and (b) temperature field history.

across the specimen. The persistence of only four fronts during unloading means the fronts must move relatively quickly to traverse the gage length, and self-cooling still decreases the specimen to as low as $18.4^{\circ} \mathrm{C}$. This causes the stress to steadily decrease during $M^{+} \rightarrow A$ transformation (unloading); whereas, the stress remains relatively constant during $A \rightarrow M^{+}$ transformation (loading) where the latent heat was distributed among twice as many (eight) fronts.

The contour maps just shown give a good overview of the thermo-mechanical behavior, but quantitative data can be somewhat difficult to see, so Fig. 13 shows selected strain profiles at 16-s intervals during the first load-unload cycle, all mapped back to the Lagrangian (reference) frame. The dashed arrows show the progression of profiles in time. (Note that, as previously discussed, the sharp oscillations at $x / L=0.13$ and 0.86 are due to the LE tags and the oscillation at $x / L=0.56$ is the artifact due to the CCD imperfection.)
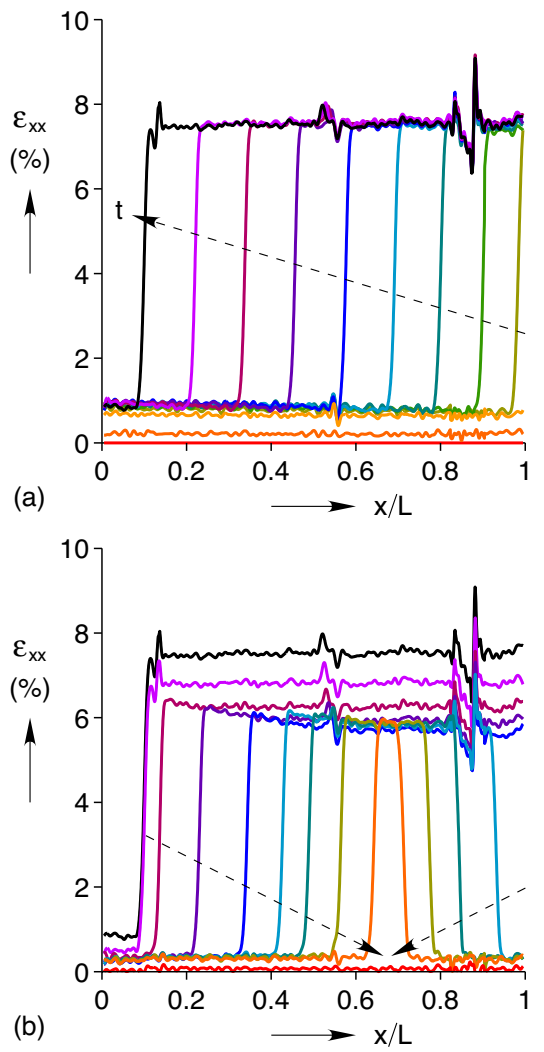

Figure 13 Experiment 2, cycle 1 axial strain profiles during loading (a) and unloading (b). Shown in the Lagrangian frame.

Lastly, Fig. 14 shows a comparison of strain $\varepsilon_{x x}$ profiles for cycles $N=1,5$, and 25 during loading at a selected strain level $\left(\delta_{\mathrm{e}} / L_{\mathrm{e}}=4.5 \%\right)$ midway across the upper stress plateau. The three strain profiles correspond to times $t=117.4 \mathrm{~s}, 1462.2 \mathrm{~s}$, and $7654.2 \mathrm{~s}$, respectively. One can again see the number of fronts (strain jumps) increases and the magnitude of the strain jumps decreases with cycle number. Comparing cycles 1 and 25 , the strain jump across a $A \rightarrow M^{+}$ transformation front during loading decreases from about $\Delta \varepsilon_{x x}=6.6 \%$ to about $3.5 \%(=6.1-2.6 \%)$, and the strain jump across a $M^{+} \rightarrow A$ transformation front during unloading (not shown) changes from $-5.7 \%$ to about $-3.5 \%(=1.5-5.0 \%)$.

\section{Summary and Conclusions}

Digital image correlation (DIC) is a modern and powerful experimental technique to characterize the evolution of deformation in structures. It is a technique that is becoming increasingly common for macroscale investigations in testing labs, and its use with optical digital cameras is now well 


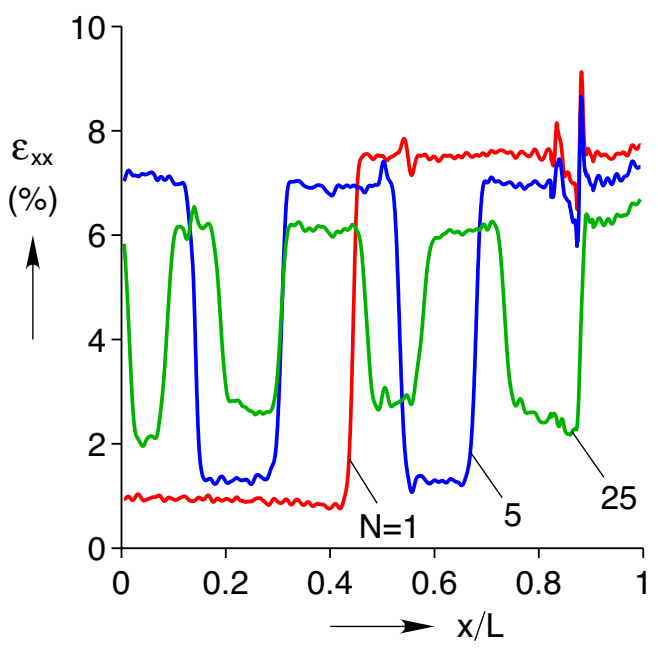

Figure 14 Experiment 2, axial strain profiles for cycles 1, 5, and 25 during loading at $\delta_{e} / L_{e}=4.5 \%$ (Lagrangian frame).

understood. DIC is appealing for many reasons, including its temporal scalability, length scalability, robust nature, and good accuracy. The wealth of quantitative information it provides on full-field surface deformations makes it an extremely useful tool in experimental mechanics. It is relatively straightforward to obtain good macroscopic results with DIC, but some attention to detail and a few tips and tricks can lead to quite accurate measurements, even on slender structures such as SMA wires.

We have demonstrated two experiments on two different types of SMA wire, where DIC has been used to quantify the nonuniform strain fields during Joule heating actuation and superelastic cyclic loading, respectively. The basics of DIC were presented along with our recommendations for specimen preparation and experimental setup to achieve accurate measurements. Even simple SMA structures, such as uniaxially loaded SMA wire, exhibit interesting and complex phenomena, and DIC has proven to be quite useful to quantify and explain the behavior.

\section{Acknowledgments}

Our thanks again go to Nilesh Mankame (General Motors) for the invitation to write this series of papers. We gratefully acknowledge the financial support for this work, provided by the National Science Foundation (CMMI-0727331) and General Motors Corp. through the GM/UM Collaborative Research Laboratory in Smart Materials and Structures.

\section{References}

1. Shaw, J.A., Churchill, C.B., and Iadicola, M.A., "Tips and Tricks for Characterizing Shape Memory Alloy Wire: Part 1 - Differential Scanning Calorimetry \& Basic Phenomena," Experimental Techniques 32(5):55-62 (2008).

2. Churchill, C.B., Shaw J.A., and Iadicola, M.A., "Tips and Tricks for Characterizing Shape Memory Alloy Wire: Part 2 - Fundamental Isothermal Responses," Experimental Techniques 33(1):51-62 (2009).

3. Churchill, C.B., Shaw, J.A., and Iadicola, M.A., "Tips and Tricks for Characterizing Shape Memory Alloy Wire: Part 3 - Localization And Propagation Phenomena," Experimental Techniques 33(5):70-78 (2009).

4. Churchill, C.B., Shaw, J.A., and Iadicola, M.A., "Tips and Tricks for Characterizing Shape Memory Alloy Wire: Part 4 - Thermo-Mechanical Coupling," Experimental Techniques 34(2):63-80 (2010).

5. Sutton, M.A., Orteu, J.J., and Schreier, H.W., Image Correlation for Shape, Motion, and Deformation Measurements: Basic Concepts, Theory, and Applications, Springer, New York (2009).

6. Daly, S., "Digital Image Correlation in Experimental Mechanics for Aerospace Materials and Structures," Encyclopedia of Aerospace Engineering, Wiley and Sons (2010).

7. Réthoré, J., Hild, F., and Roux, S., "Extended Digital Image Correlation with Crack Shape Optimization," International Journal for Numerical Methods in Engineering 73:248-272 (2008).

8. Poissant, J., and Barthelat, F., "A Novel "Subset Splitting" Procedure for Digital Image Correlation on Discontinuous Displacement Fields," Experimental Mechanics 50:353-364 (2010). DOI: 10.1007/s1 1340-009-9220-2.

9. Peters, W.H., and Ranson, W.F., “Digital Imaging Techniques in Experimental Stress Analysis," Optical Engineering 21(3):427-432 (1982).

10. Sutton, M.A., Wolters, W.J., Peters, W.H., Ranson, W.F., and McNeill, S.R., "Determination of Displacements Using an Improved Digital Correlation Method," Image and Vision Computing 1(3):133-139 (1983).

11. Peters, W.H., Ranson, W.F., Sutton, M.A., Chu, T.C., and Anderson, J., “Application of Digital Correlation Methods to Rigid Body Mechanics," Optical Engineering 22:738-742 (1983).

12. Luo, P., Chao, Y., Sutton, M., and Peters, W., “Accurate Measurement of Three-dimensional Deformations in Deformable and Rigid Bodies Using Computer Vision," Experimental Mechanics 33(2):123-132 (1993).

13. Faugeras O., Three-Dimensional Computer Vision: A Geometric Viewpoint, MIT Press, Cambridge, MA (1993). 
14. Helm, J.D., McNeill, S.R., and Sutton, M.A., "Improved Three-dimensional Image Correlation for Surface Displacement Measurement," Optical Engineering 35:1911-1920 (1996).

15. Bay, B., Smith, T., Fyhrie, D., and Saad, M., "Digital Volume Correlation: Three-dimensional Strain Mapping Using X-ray Tomography," Experimental Mechanics 39:217-226 (1999). DOI: 10.1007/BF02323555.

16. Franck, C., Hong, S., Maskarinec, S., Tirrell, D., and Ravichandran, G., “Three-dimensional Full-field Measurements of Large Deformations in Soft Materials Using Confocal Microscopy and Digital Volume Correlation," Experimental Mechanics 47:427-438 (2007). DOI: 10.1007/s1 1340-007-9037-9.

17. Sutton, M.A., McNeill, S.R., Helm, J.D., and Chao, Y.J., "Advances in Two-dimensional and Three-dimensional Computer Vision," Photomechanics, Topics Applied Physics, Volume 77, Springer Verlag, pp. 323-372 (2000).

18. Schreier, H.W., Garcia, D., and Sutton M.A. "Advances in Light Microscope Stereo Vision," Experimental Mechanics 44:278-288 (2004).

19. Schreier, H.W., Braasch, J.R., and Sutton, M.A., "Systematic Errors in Digital Image Correlation Caused by Intensity Interpolation," Optical Engineering 39(11):2915-2921 (2000).

20. Sutton, M.A., Yan, J.H., Tiwari, V. Schreier, H.W., and Orteu, J.J. "The Effect of Out-of-plane Motion on 2D and 3D Digital Image Correlation Measurements," Optics and Lasers in Engineering 46(10):746-757 (2008).

21. Zavattieri, P.D., Savic, V. Hector, L.G., Jr., Fekete, J.R., Tong, W., and Xuan, Y., "Spatio-temporal Characteristics of the Portevin-Le Châtelier Effect in Austenitic Steel with Twinning Induced Plasticity," International Journal of Plasticity 25:2298-2330 (2009).

22. Knauss, W.G., Chasiotis, I., and Huang, Y., "Mechanical Measurements at the Micron and Nanometer Scales," Mechanics of Materials 35:217-231 (2003).

23. Chu, T.C., Ranson, W.F., and Sutton, M.A. "Applications of Digital-image-correlation Techniques to Experimental Mechanics," Experimental Mechanics 25(3):232-244 (1985).

24. Reu, P.L., and Miller, T.J., "The Application of High-speed Digital Image Correlation," The Journal of Strain Analysis for Engineering Design 43(8):673-688 (2008).

25. Berfield, T., Patel, J., Shimmin, R., Braun, P., Lambros, J., and Sottos, N., "Micro- and Nanoscale
Deformation Measurement of Surface and Internal Planes via Digital Image Correlation," Experimental Mechanics 47(1):51-62 (2007).

26. Churchill, C.B., and Shaw, J.A., "Thermo-electro-mechanical Shakedown Response of Conditioned Shape Memory Alloy Wires," Proceedings of the ASME 2009 Conference on Smart Materials, Adaptive Structures and Intelligent Systems (SMASIS 2009), ASME, Volume 1, pp. 137-148, (2009).

27. National Instruments (www.dasylab.com), Data Acquisition System Laboratory (DASY-Lab) Manual, DASY-Lab, Austin, TX.

28. Tong, W. "A Users Guide to the Yale Surface Deformation Mapping Program (SDMAP)," Technical Report, Department of Mechanical Engineering, Yale University, New Haven, CT (2004).

29. Tong, W. “An Evaluation of Digital Image Correlation Criteria for Strain Mapping Applications," Strain 41(4):167-175 (2005).

30. Sutton, M.A., McNeill, S.R., Jang, J., and Babai, M. "Effect of Subpixel Image Restoration on Digital Correlation Error Estimates," Optical Engineering 1988:870-877 (1988).

31. Bruck, H., McNeill, S. Sutton, M., and Peters, W., "Digital Image Correlation Using Newton-Raphson Method of Partial Differential Correction," Experimental Mechanics 29(3):261-267 (1989).

32. Vendroux, G., and Knauss, W.G., "Submicron Deformation Field Measurements: Part 2. Improved Digital Image Correlation," Experimental Mechanics 38:86-92 (1998).

33. Iadicola, M.A., and Shaw, J.A., "The Effect Of Uniaxial Cyclic Deformation on the Evolution of Phase Transformation Fronts in Pseudoelastic NiTi Wire," Journal of Intelligent Material Systems and Structures 13(2):143-156 (2002).

34. Sun, H., Pathak, A., Luntz, J., Brei, D., Alexander, P.W., and Johnson, N.L., "Stabilizing Shape Memory Alloy Actuator Performance Through Cyclic Shakedown: An Empirical Study," Porter Davis, L., Henderson, B.K., and McMickell, M.B., (eds), Proceedings of SPIE, Industrial and Commercial Applications of Smart Structures Technologies 2008, Volume 6930, SPIE, pp. 69300Q-(2008).

35. Correlated Solutions, Inc. (www.correlatedsolutions.com), Vic-Snap Manual, Correlated Solutions, Inc., Columbia, SC.

36. Correlated Solutions, Inc. (www.correlatedsolutions.com), Vic-3D Manual, Correlated Solutions, Inc., Columbia, SC. 